\title{
Labyrinthe
}

23 | 2006 (1)

Quatre chercheurs de l'étranger

\section{La science ouverte d'Enrico Castelli Gattinara}

David Schreiber

\section{OpenEdition}

Journals

Édition électronique

URL : http://journals.openedition.org/labyrinthe/1162

DOI : $10.4000 /$ labyrinthe. 1162

ISSN : 1950-6031

Éditeur

Hermann

Édition imprimée

Date de publication : 15 janvier 2006

ISBN : 2-9526131-0-9

Référence électronique

David Schreiber, « La science ouverte d'Enrico Castelli Gattinara », Labyrinthe [En ligne], 23 | 2006 (1), mis en ligne le 24 juillet 2008, consulté le 08 mai 2019. URL : http://journals.openedition.org/ labyrinthe/1162 ; DOI : 10.4000/labyrinthe.1162

Propriété intellectuelle 


\section{La science ouverte d'Enrico Castelli Gattinara}

David SCHREIBER

Enrico Castelli Gattinara n'est un philosophe «étranger» que pour moitié. «Philosophe de 45 ans», comme on devrait parler aujourd'hui, deux siècles après Balzac, de la «femme de 45 ans », il appartient à cette catégorie de chercheurs qui possèdent une véritable double culture et qui savent la mettre à profit. Italien, laureato de philosophie en 1983, il a soutenu une thèse à l'École des hautes études en sciences sociales à Paris en 1992. Une version de celle-ci a été publiée chez Vrin en 1998 sous le titre Les Inquiétudes de la raison, épistémologie et histoire en France dans l'entre-deux-guerres, et l'on pourra aussi lire les deux articles en français publiés dans le $119^{\mathrm{e}}$ tome de la Revue de synthèse sur les rapports entre épistémologie, histoire et histoire des sciences. Enrico Castelli Gattinara enseigne aujourd'hui l'épistémologie de l'histoire à l'université La Sapienza de Rome mais continue de venir animer divers séminaires à Paris. S'il a traduit en 1995 La Formation de l'esprit scientifique de Bachelard en italien, que l'on ne se méprenne pas: ce n'est pas en tant que passeur italien d'une pensée française sur la science que nous avons le plaisir de le publier ici. Il y aurait là une drôle de manière de s'intéresser à des pensées autres! Mais ce n'est pas non plus en tant que «bon sauvage», ultime «joker» d'une communauté de chercheurs en mal de références stratégiques à faire valoir sur le marché des académismes divers. C'est d'abord parce que son travail propose une série de positions philosophiques dont la pertinence nous paraît forte. Ainsi la reprise du «moment 1900-1930» dans l'histoire des savoirs, plus particulièrement en France, a permis à Castelli Gattinara de montrer toute l'importance de l'histoire comme mode de compréhension des procédures mêmes du travail des sciences sur elles-mêmes, en particulier pour tout projet épistémologique. Affaire entendue, diront certains, mais qui vaut la peine d'être rappelée face à la déferlante des prises de position très récentes, qui, loin d'ouvrir vers une réelle appréhension du phénomène de l'his- 
toire des sciences, tend plutôt à rigidifier les argumentations, y compris dans leur versant «sociologique». En ce sens, Castelli Gattinara a rappelé que les années 1900-1930 sont à prendre à la fois comme moment de rupture du point de vue de l'appréhension des phénomènes scientifiques, mais aussi comme moment intellectuel où diverses tentatives, assez hétérogènes lorsqu'on y regarde de près, assez peu coordonnées aussi, cherchent à rendre compte de cette rupture et à la prendre comme le fait de la science plutôt que comme un écart par rapport à ce qu'elle devrait être. On rappellera ici l'importance des travaux d'un Brunschvicg, d'un Duhem, d'un Bachelard, d'un Koyré ou d'un Lenoble, directement en prise avec cette rupture et dont les pensées sont déterminantes pour Castelli Gattinara. Moment fondamental, donc, où «au nom du rationalisme et de sa sauvegarde dans la crise» s'opèrent un échange et une reconfiguration réciproque de l'épistémologie et de l'histoire (Les Inquiétudes de la raison, p. 308).

Dès lors, le pas est franchi vers une théorie de la création scientifique comme temps «sublime», que le texte présent cherche à dégager. L' «inquiétude» fait place à l'émotion devant l'expérience. Le sublime kantien permet d'engager la réflexion sur l'ouverture de la science vers d'autres formes de l'activité intellectuelle, l'art, la mémoire, le langage. De tout cela, il ressort une confiance dans la construction du savoir comme activité de croisement, de superposition, ou d' «intersection» - une notion à laquelle le philosophe est très attaché. Nulle histoire linéaire et cumulative de la science, dont on pourrait décrire en surplomb les efforts de normalisation, de dépassement, d'englobement mais bien une histoire contigente, faite de déplacements, de ruptures, d'oublis et de rappels. Mode réel de la pensée de «l'impensable», qui permet à la science d'approcher sans cesse le monde, de se construire sur un passé et d'avoir un avenir, et dont le philosophe peut rendre raison, sans défaitisme, avec les armes mêmes de l'expérience (Pensare l'impensato est le titre de son dernier livre italien, paru en 2004).

Mentionnons pour finir qu'Enrico Castelli Gattinara est aussi directeur de la revue italienne Aperture (http://digilander.libero.it/aperture). Créée en 1996, cette grande sœur italienne de Labyrinthe s'appuie sur le même constat et vise le même objectif: partir du fait admis de la pluralité des sphères du travail intellectuel et des références culturelles, et viser néanmoins à «ouvrir» ces univers les uns sur les autres, à renforcer le débat. 